\title{
Disconnection and reconnection: the morphological basis of (mal)adaptation to stress
}

\author{
Nuno Sousa ${ }^{1,2}$ and Osborne F.X. Almeida ${ }^{1,2,3}$ \\ ${ }^{1}$ Life and Health Science Research Institute (ICVS), School of Health Sciences, University of Minho, Braga, Portugal \\ ${ }^{2}$ ICVS/3B's - PT Government Associate Laboratory, Braga/Guimarães, Portugal \\ ${ }^{3}$ Max-Planck Institute for Psychiatry, Munich, Germany
}

\begin{abstract}
Maladaptive responses to stress and the associated hypersecretion of glucocorticoids cause psychopathologies ranging from hyperemotional states and mood dysfunction to cognitive impairments. Research in both humans and animal models has begun to identify morphological correlates of these functional changes. These include dendritic and synaptic reorganization, glial remodeling, and altered cell fate in cortical and subcortical structures. The emerging view is that stress induces a 'disconnection syndrome' whereby the transmission and integration of information that are critical for orchestrating appropriate physiological and behavioral responses are perturbed. High-resolution spatiotemporal mapping of the complete neural circuitry and identification of the cellular processes impacted by stress will help to advance discovery of strategies to reduce or reverse the burden of stress-related neuropsychiatric disorders.
\end{abstract}

\section{Introduction}

Hans Selye's so-called general adaptation syndrome embodies the idea that survival depends on an organism's ability to sense and make appropriate physiological and behavioral responses to potentially threatening stimuli of internal and external origin [1]. Whereas glucocorticoids (GCs), secreted by the adrenal glands, play an important role in reinstating homeostasis during and after stress (allostasis) [2], coordination of the adaptive response depends on the brain [3]. The brain not only perceives (alarm phase) and assigns value to potential threats, but also plans and executes cost-effective defense strategies (resistance phase); the latter includes homeostatic regulation of GC secretion [3]. By contrast, the brain can fall victim to stressors that occur in specific contexts or that exceed a certain intensity and/or duration, leading to exhaustion [2]. When this happens, physiological and behavioral homeostasis deteriorates, rendering the individual vulnerable to the cumulative burden of allostatic load (maladaptive responses) that ultimately triggers somatic and neuropsychiatric disorders [2]. The best examples of the latter are mild to severe cognitive impairment, anxiety and mood disturbances, drug and substance abuse, and eating

Corresponding author: Sousa, N. (njcsousa@ecsaude.uminho.pt). Keywords: stress; cognition; emotion; plasticity; glucocorticoids. disorders [4]. After consideration of the impact of stress on the fate (proliferation and survival) and structure and function (synaptic transmission) of neural cells in areas implicated in some of the aforementioned brain disorders, we arrive at the conclusion that stress disrupts normal brain functioning by interrupting connectivity between different brain regions.

\section{Stress regulates neural cell fate}

The suggestion that chronic stress disrupts brain structure and function by triggering a vicious cycle that results in unabated secretion of GCs proved to be both stimulating and provocative [5]. GC hypersecretion was ascribed to a loss of corticosteroid receptor-bearing hippocampal neurons [6], resulting in a loss of corticosteroid feedback inhibition. This view was strengthened by subsequent observations that GCs can directly target hippocampal neurons for apoptosis [7]. GCs are also known to increase $\mathrm{Ca}^{2+}$ influx [8], thus rendering neurons more vulnerable to excitotoxicity $[9,10]$ and oxidative stress [11]. Causal links between high GC levels and hippocampal degeneration have also become evident from magnetic resonance imaging (MRI) studies in humans. For example, hippocampal atrophy accompanies two conditions in which GC hypersecretion is a key pathological factor: Cushing's syndrome [12] and recurrent major depression [13]. Notably, cognitive improvements are seen after remedy of the hypercortisolismic state in Cushingoid subjects [14], and two studies reported trends towards increased hippocampal volumes in depressed patients who received antidepressants over an extended period [15] and in patients who showed reduced GC secretion in response to antidepressant therapy [16]. By contrast, reports that hippocampal cell numbers are not altered after chronic exposure of rats to elevated GC levels [17] have raised questions regarding the functional importance of GC-induced hippocampal cell death.

The discovery that new neurons can be generated throughout life in some brain regions, including the hippocampal dentate gyrus, introduced a new variable into the equation concerning regulation of brain structure and functional integrity by stress. Newly generated neurons not only integrate into the preexisting hippocampal circuitry $[18,19]$ but also facilitate the formation of new 
memories [18-20], presumably by influencing the activity of other circuits with which the hippocampus shares reciprocal connections. This is exemplified by the finding that hippocampal neurogenesis is essential for manifestation of the anxiolytic actions of antidepressant drugs in rats [21,22]; it should be noted that anxiety is regulated by the bed nucleus of the stria terminalis (BNST), an extension of the amygdala that receives modulatory inputs from the hippocampus.

The so-called neurogenic hypothesis of depression receives support from observations that hippocampal neurogenesis is markedly attenuated in animal models of depression [21-23]; most of these models recapitulate the GC hypersecretion seen in a substantial proportion of depressed patients [24,25]. Given that most antidepressants stimulate neurogenesis [21], it is surprising to note that two independent studies concluded that neurogenesis does not appear to be critical for manifestation of the initial therapeutic response to antidepressants in rodents, as judged by two surrogate markers of the disease, learned helplessness and anhedonia [21,22]. Interestingly, however, hippocampal neurogenesis facilitates restoration of normal levels of GC secretion after stress in rodents $[26,27]$, suggesting that neurogenesis might be important in remission phases of the disease. In addition, evidence that (i) the modulatory actions of newly generated hippocampal cells may prevail over mature hippocampal neurons $[18,28,29]$ and (ii) recently produced neurons facilitate memory allocation and consolidation and thus improve memory retention [30] suggests that long-term recovery from depression may depend on the capacity to regain neurogenic potential. Within the general framework of this article, the finding that GCs specifically target neuronal progenitors for apoptosis [31] deserves mention.

It was recently shown that astroglial cells are sensitive to the effects of stress. Specifically, chronic stress inhibited the proliferation of glial progenitor cells in the prefrontal cortex (PFC) and hippocampus [32,33] and decreased the number of glial-fibrillary acidic protein (GFAP)-positive cells and expression of GFAP in the prelimbic cortex [34] in rodents. Given that a single astrocyte serves thousands of synapses, the ramifications of such alterations for the optimal function of neuronal networks could be significant [35]. Glial cells are crucial members of the glutamatergic tripartite synapse, where they serve to maintain synaptic homeostasis. Changes in glial cell numbers or astrocytic remodeling may promote glutamate spillover to extrasynaptic sites [35], resulting in activation of extrasynaptic glutamate receptors and promotion of excitoxicity [36]; as previously mentioned, GCs may exacerbate the latter [10]. Interestingly, post mortem examination of subjects with stress-related mood disorders has revealed marked reductions in the number and density of glial cells in the PFC [37].

\section{Stress as a disconnecting syndrome}

Although gains and losses in neuronal and glial numbers provide a first level of modulation of neuronal networks, their occurrence is relatively rare except in cataclysmic events such as stroke. More commonly, functional plasticity in the brain is preceded by structural plasticity, typically in the form of synaptic remodeling of neurons and astroglia; this remodeling may include alterations in the number of dendrites and the type of spines (Box 1). The idea that GCs may impair brain function by compromising

\section{Box 1. Stress-induced changes in spine plasticity}

Modern microscopy methods and associated algorithms permit a comprehensive neuronal morphology analysis, from 3D dendritic reconstruction to estimation of spine numbers and densities, as well as categorization of spines based on morphological characteristics. Distinction between immature (thin and stubby) and mature (mushroom, wide and ramified) spines is important because they serve different functions [21]. Thin and stubby spines are most commonly found during development and are thought to represent immature plastic structures, whereas wide and ramified mushroom spines are more stable and represent stronger synapses. Accordingly, shifts in spine morphology are increasingly used to describe synaptic plasticity induced by stress and other stimuli.

Stress affects spine density and morphology in a brain regionspecific manner. In the PFC and hippocampus [38,39,57-64], chronic stress triggers dendritic atrophy and reductions in spine numbers (Figure la), with a parallel increase in the relative number of immature spines. However, in other brain regions (e.g., orbitofrontal cortex, amygdala and BNST), repeated stress results in increased dendritic length and spine density (Figure lb) $[90,101,112]$.

The ability to compile information about changes in spine shape and function with high spatiotemporal resolution provides greater insights not only into the dynamic reorganization of postsynaptic function during stress but also into the disconnection syndrome that may underlie stress-related diseases of the brain.

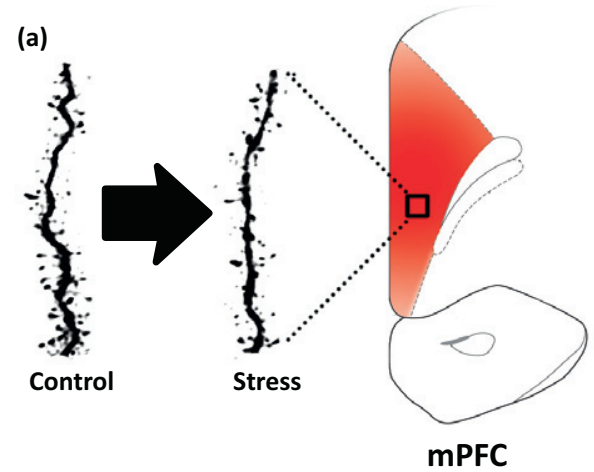

(b)

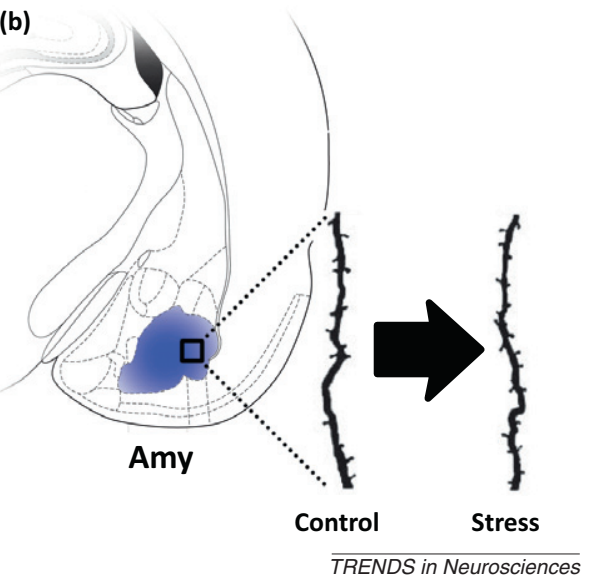

Figure I. Schematic representation of the contrasting effects of chronic stress on dendritic spine numbers in different brain regions. (a) A decrease in the number of spines has been reported in pyramidal neurons of the infralimbic cortex of rats after repeated restraint stress (21 days) [61]. (b) By contrast, chronic immobilization stress (10 days) triggers an increase in the number of spines in basolateral amygdala spiny neurons in rats [101]. Adapted, with permission, from (a) [61] and (b) [101]. Abbreviations: Amy, amygdala; mPFC, medial prefrontal cortex. 
neuronal plasticity stems from reports that chronically high GC levels impair cognitive performance and trigger dendritic atrophy and synaptic loss in certain subpopulations of hippocampal neurons [38,39]. Importantly, the latter changes are reversible: when previously stressed rats are allowed an intervening stress-free period, the hippocampus displays a recovery of dendritic arbors and synaptic numbers, accompanied by reversal of the stressinduced impairments in hippocampus-dependent memory [39]. These morphological adaptations may explain the increased hippocampal volumes and cognitive improvements seen after surgical correction of Cushing's syndrome in humans [14]. Such transient effects of GCs on the structure and function of the hippocampus, as well as other brain regions, tend to support the view that dendritic atrophy, rather than neuronal elimination, is the key mechanism through which high GC levels interfere with connectivity patterns in dynamic neuronal networks.

Until recently, research on the effects of stress has mostly been concentrated on the hippocampus because of its abundant expression of corticosteroid receptors $[40,41]$ but there is now growing interest in generating systematic maps of stress-responsive circuits in the brain as a whole [2]. These new efforts include focus on the classic slow actions triggered by corticosteroids and on the novel fast actions of corticosteroid receptors and their intermediate pathways, which contribute to a better understanding of the events that occur from the perception of stress to the adaptive neuroendocrine and behavioral responses [42]. The emerging picture is that stress-responsive areas in the brain are organized into functionally integrated networks [43-45] whose main components include the hippocampus, brainstem nuclei (locus coeruleus and raphé), hypothalamus, extended amygdala, dorsal and ventral striatum, medial $\mathrm{PFC}(\mathrm{mPFC})$, and orbitofrontal cortex (OFC) (Figure 1). Such interconnections are likely to explain why the disruptive effects of stress are generally not restricted to a single function. For example, stress initially interferes with hippocampus-dependent declarative memory but eventually also impairs frontocorticaldependent cognitive functions (e.g., working memory, behavior flexibility, and decision-making), as well as behavioral domains that are regulated by multiple brain areas (e.g., mood, anxiety, fear) [46,47], as discussed later.

The PFC, and in particular the $\mathrm{mPFC}$, has attracted much attention from researchers interested in the neurobiology of stress because of its crucial role in the regulation of executive and cognitive functions [48] and its implication in stress-related psychiatric disorders [49]. Both the hippocampus and $\mathrm{mPFC}$ express corticosteroid receptors $[40,41,50]$ and thus contribute to coordination of the initial and terminal phases of the stress response [51-53]. Loss of (a)

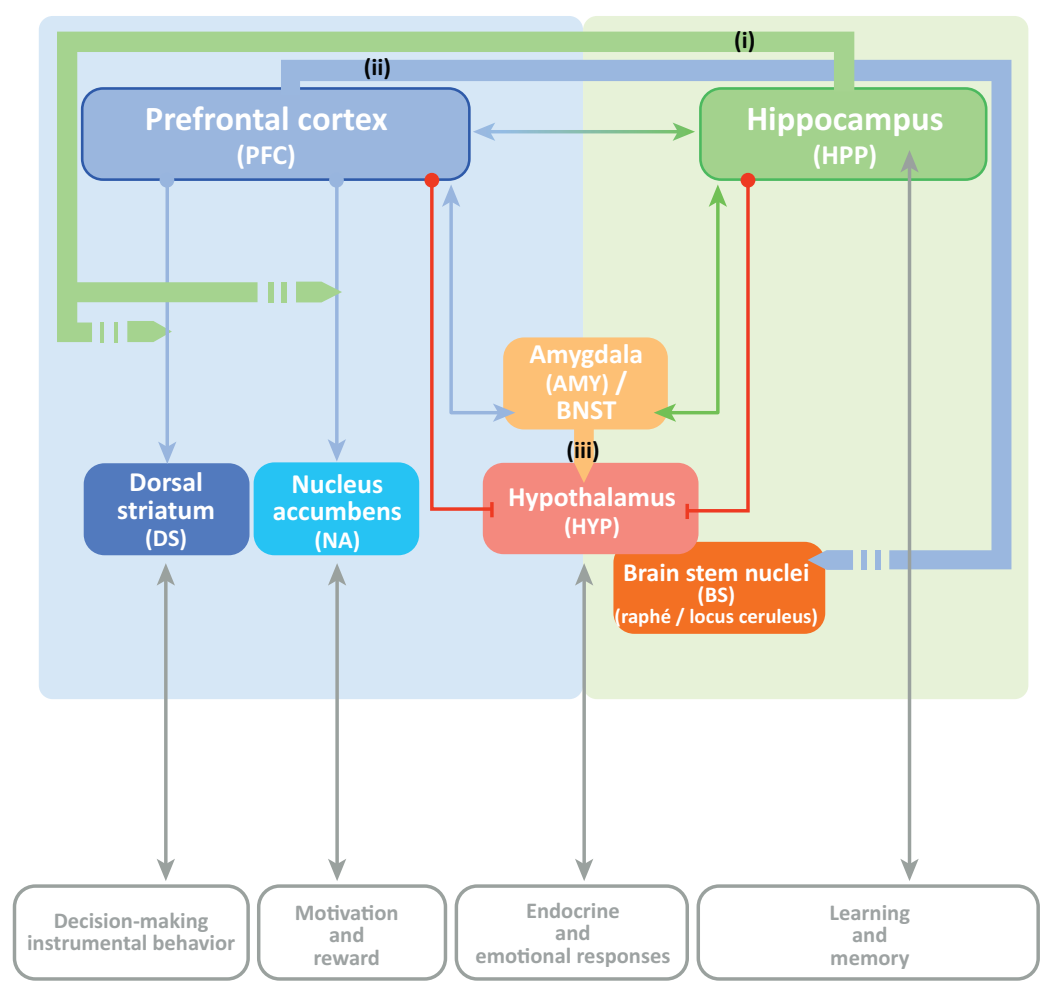

(b)
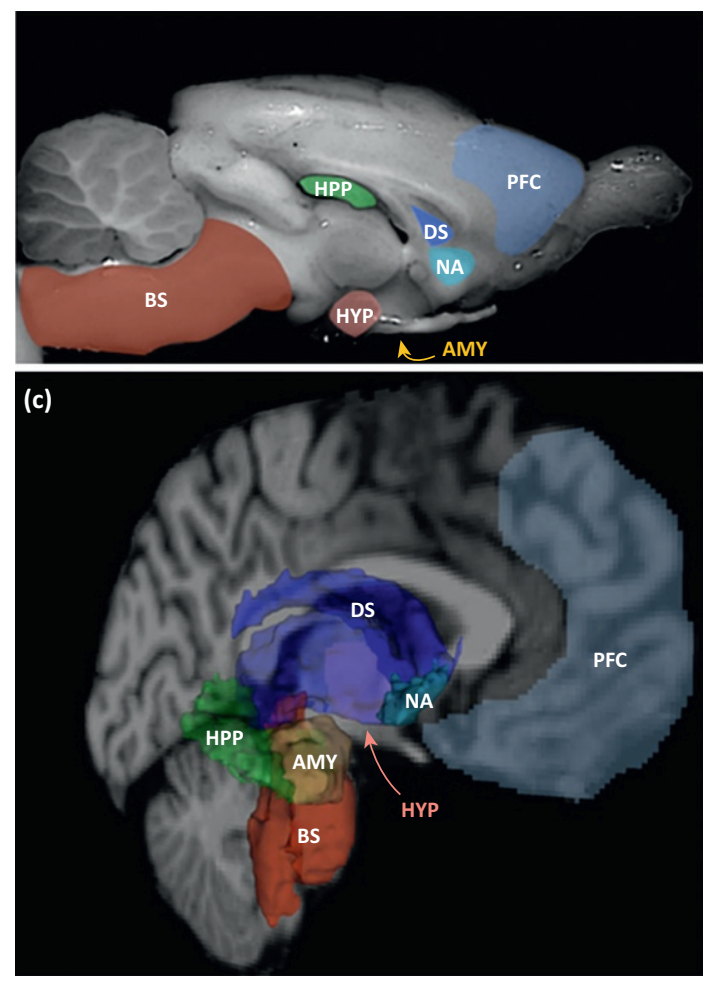

$\overline{\text { TRENDS in Neurosciences }}$

Figure 1. Limbic-cortical circuits targeted by stress and involved in coordinating the endocrine and behavioral responses to stress. (a) The prefrontal cortex (PFC) and hippocampus (HPP) play a central integrative role in the response to stress. The hippocampal formation is mainly involved in integration of sensory-cognitive information received from the PFC and amygdala (green background and arrows); it is also implicated in contextual gating (i) as it modulates the flow of information from the PFC to the striatum, including the nucleus accumbens (NA). The PFC regulates emotional and cognitive functions (ii) through its modulation of hippocampal and subcortical activity, including dorsal striatum (DS) and brain stem (BS) nuclei activity (blue background and arrows); it plays a critical role in decision-making processes, instrumental behavior motivation, and reward. In addition, both the PFC and HPP contribute to inhibitory control of the paraventricular nucleus of the hypothalamus (HYP) (red arrows), and thus to control of the hypothalamic-pituitary-adrenal axis (HPA) axis and modulation of the activity of brain stem nuclei. The amygdala and bed nucleus of stria terminalis (BNST) provide excitatory input to the HYP and thus participate in regulation of autonomic functions (iii). The influence of these brain regions in different behaviors and responses affected by stress is also highlighted (grey arrows). (b,c) Schematic representations of the topography of these regions in the (b) rodent and (c) human brain. 
corticosteroid receptors in the hippocampus [5] and $\mathrm{mPFC}$ [54] in response to chronic stress breaks the GC negative feedback loop, leading to persistently elevated GC secretion.

Both chronic stress and treatment with exogenous GCs reduce the volume of the superficial layers of the rodent $\mathrm{mPFC}[55,56]$. These reductions reflect alterations in the morphology of layer II/III [57-63] and layer V [64] pyramidal neurons: stress and GCs cause shrinkage of dendritic trees, especially in the right prelimbic and infralimbic $[65,66]$ and left anterior cingulate cortex [65]. These observations match those found in neuroimaging studies in rodents [56] and humans [67] in which high GC levels are associated with reduced size of the left cingulate cortex. The ramifications of all these observations could be significant in the context of laterality and brain function in health and disease because the left PFC plays a key role in knowledge-intensive reasoning and decision-making processes $[68,69]$, whereas the right $\mathrm{PFC}$ is primarily responsible for perceptual reorganization [70] and conflict detection and resolution [69,71]. It is also interesting to note that functional neuroimaging has revealed unambiguous left-right shifts in the activity of cortical and subcortical structures during depressive episodes and following their pharmacological alleviation [72,73].
The importance of recognizing that stress induces impairments in brain function in a spatiotemporal and stepwise fashion is well illustrated by considering how stress independently and co-dependently disrupts behaviors that require either an intact hippocampus or $\mathrm{PFC}$, or both (Figure 2). A previous study demonstrated that whereas acute stress (3 days) in rats only impairs spatial reference memory, chronic stress (28 days) produces impairments in both spatial working memory and behavioral flexibility (Figure 2a-c) [74]. On the basis of knowledge that (i) behavioral flexibility - critical for facilitating adaptation to fluctuations in environmental conditions depends on the integrity of the PFC; (ii) spatial reference memory is a hippocampus-dependent function; and (iii) spatial working memory requires intact connections between the hippocampus and PFC, these findings point to the greater sensitivity and/or earlier response of the hippocampus in these experimental conditions to the deleterious actions of stress. It should be noted, however, that other studies have revealed that the effects of stress on spine and dendritic morphology in the apical trees of $\mathrm{mPFC}$ pyramidal neurons - namely in the infralimbic cortex may be observed after short exposure to stress [75,76]. Importantly, these structural changes were accompanied by resistance to fear extinction (Figure 3) [76], although it

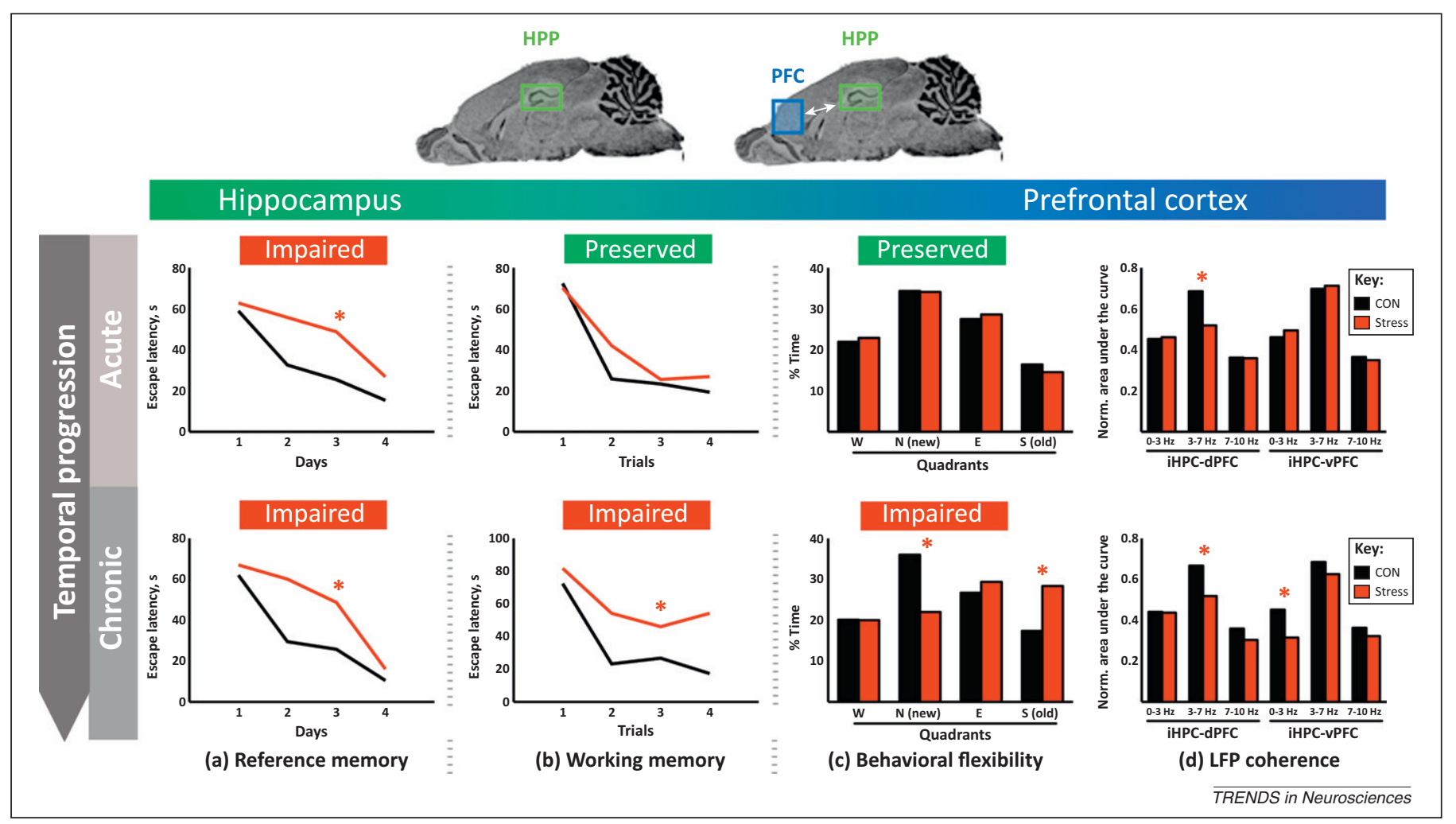

Figure 2. Schematic representation of stress-induced behavioral and electrophysiological effects in the hippocampal-prefrontal cortical (PFC) network in the rat. (a)

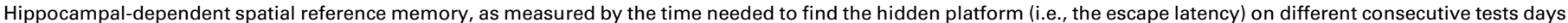

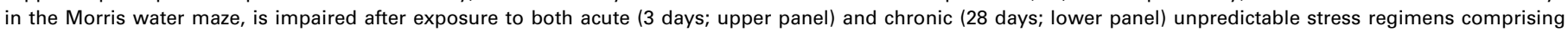
multiple stressors delivered in an unpredictable order and time of day. (b) Spatial working memory, measured as the mean escape latency time within trials in the water maze, is impaired after chronic stress ( 28 days; lower panel) but not after exposure to acute stress ( 3 days; upper panel). Such a memory task depends on intact

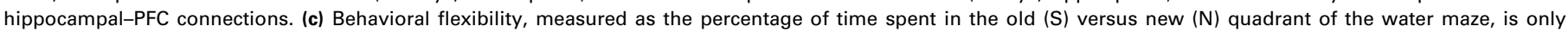
damaged after prolonged stress ( 28 days; lower panel) and not after acute stress ( 3 days; upper panel). This task depends on intact PFC structures. (d) Local field potential (LFP) coherence in lower-frequency oscillations $(<10 \mathrm{~Hz})$ between the hippocampus and PFC is differentially affected by acute ( 3 days) versus chronic (14 days) stress, whereas acute stress impairs coherence between the hippocampus (HPC) and dorsal PFC (dPFC; upper panel) in the theta frequency (3-7 Hz). Prolonged stress exposure also leads to a loss of coherence between the HPC and ventral PFC (vPFC) in the delta frequency. In all panels, responses by animals subjected to stress (Stress) are shown in red; responses by animals under control conditions (CON) are shown in black. Asterisks denote statistically significant changes. Adapted, with permission, from (a-c) [74] and (d) [78]. 


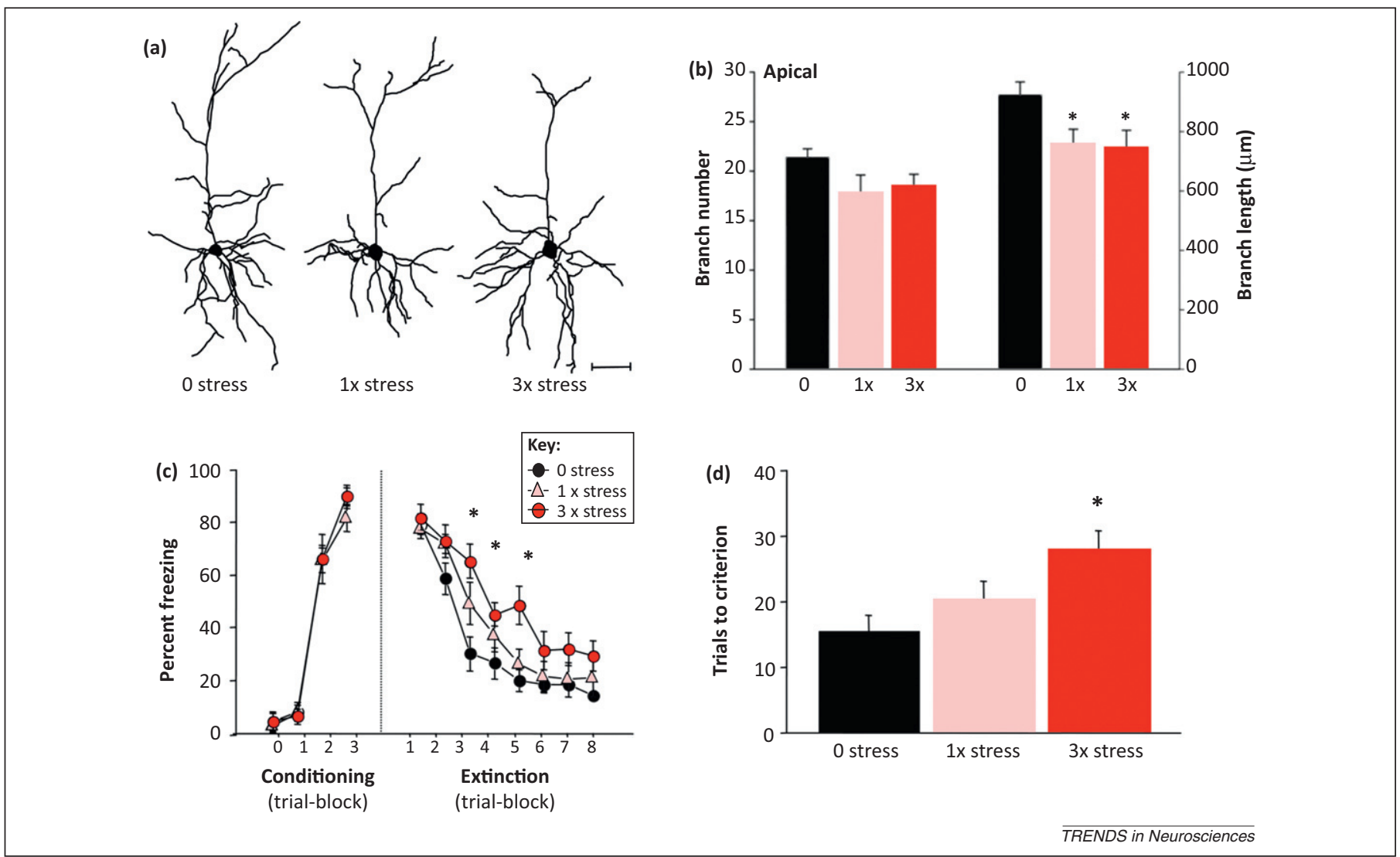

Figure 3. Schematic representation of acute stress-induced structural effects in the infralimbic (IL) cortex of mice and its behavioral correlates in fear extinction. (a) Computer-assisted reconstructions of representative IL pyramidal neurons in mice exposed to zero $(0)$, one $(1 \times)$, or three $(3 \times)$ episodes of stress. (b) Apical dendritic branch length, but not number, were significantly reduced after one $(1 \times)$ or three $(3 \times)$ stress episodes relative to controls $(0)$. (c) Stress significantly increased freezing during extinction but did not affect fear acquisition or initial recall of the conditioned tone. (d) Three $(3 \times)$ stress episodes significantly increased the number of trials to extinction criterion. Asterisks denote statistically significant changes. Adapted, with permission, from [76].

was not reported whether working memory was affected in this study. In line with the view that stress may result in alterations in brain networks in a spatiotemporal pattern, recent studies in rats showed that stress initiates Alzheimer's disease-like pathology (i.e., overproduction of amyloid $\beta$ and abnormal hyperphosphorylation of tau protein) in the hippocampal and parahippocampal regions, from where it spreads to frontocortical areas [77].

In vivo electrophysiological studies have shown that stress disrupts hippocampal innervation of the mPFC. Stress impairs long-term potentiation (LTP) and therefore synaptic plasticity in the hippocampal-PFC pathway [74]. Furthermore, stress decreases coherence, especially of the theta band, between the hippocampus and PFC (Figure 2d) [78]; theta oscillations between these two brain regions are considered crucial for performance in cognitive tasks such as spatial working memory and long-term memory $[79,80]$. More recently, it was shown that stress-induced alterations of neuronal activity in the MPFC interfere with the retrieval of fear extinction [81]. These changes may thus be considered to be functional correlates of the aforementioned dendritic and synaptic rearrangements in afferents to the mPFC [55,60,74]. Notably, similar observations were described in the amygdala-to-PFC projection [62], highlighting the broad but specific effects of stress on neuronal networks. Resembling the observations in the hippocampus [39], stress-induced changes in PFC morphology are reversible when (young) animals are allowed a post-stress period of recovery $[64,82,83]$. Interestingly, this spontaneous recovery of plasticity can be augmented by antidepressant drugs [21], a finding that may be relevant in the context of pharmacological interventions for the management of stress-induced disorders in which impaired neuroplasticity is implicated [84].

As in other forms of plasticity, glutamatergic transmission is likely to mediate the dendritic and synaptic rearrangements that follow exposure to stress (Box 2), as well the phenomena that underlie the prevention and recovery from stress [49]. This is supported by recent data demonstrating the importance of GCs in AMPA receptor (AMPAR) and NMDA receptor (NMDAR) trafficking $[85,86]$ and of NMDARs in stress-induced dendritic atrophy [87]. Thus, GCs are probably key mediators of allostasis, a process in which their effects are integrated with the actions of a variety of other signaling molecules (e.g., catecholamines, glutamate, corticotrophin-releasing hormone, arginine vasopressin, brain-derived neurotrophic factor, and cell adhesion molecules) to produce an orchestrated adaptive response. By contrast, imbalance in any of these mediators will result in a maladaptive response (increased allostatic load).

\section{An extended view of stress: from decision-making to emotions}

As already mentioned, the stress response facilitates successful adaptation. This necessarily involves recruitment 


\section{Box 2. Stress and synaptic plasticity}

Synaptic activity is modulated by stress in both the short and longterm. Acute stress leads to increased glutamate release at the tripartite synapse, comprising a presynaptic axon, astroglial processes, and a post-synaptic neuron, increased expression of postsynaptic ionotropic glutamate receptors, and synaptic potentiation [42]. The cellular and molecular mechanisms that may account for the initial neural responses to stress, including altered cognitive functions (e.g., associative learning and working memory) remain unclear, but integration of GC-elicited signals elicited at the neuronal membrane with GC-triggered alterations in transcription (mediated by the nuclear receptors NR3C1 and NR3C2) appears to be highly likely $[42,112-115]$. It should be noted that different brain regions express differing ratios of NR3C1 and NR3C2 [40,41].

As might be expected, conditions of chronic stress involve recruitment and integration of different adaptive mechanisms. Most notable are reductions in the expression of NMDARs and AMPARs at the neuronal surface in association with altered transmission efficiency and synaptic plasticity $[86,87]$; the latter has been most commonly assessed in terms of inducibility of LTP (impaired) and long-term depression (LTD; facilitated). There is also evidence that chronic stress influences glial cell morphology, metabolism, and function, resulting in a decreased ability for glial clearance of extracellular glutamate and subsequent activation of extrasynaptic glutamatergic receptors [49]. Together, these morphological, neurochemical, and electrophysiological changes are thought to translate into impairments in spatial and contextual memory performance and attentional control. of collateral integrative mechanisms to assess and interpret information impinging on multiple sensors, rheostatic mechanisms, and appropriate neurobehavioral and physiological responses. Investigations that extend beyond the hippocampal-PFC pathway are beginning to reveal how stress also impacts on the representation, planning, and execution of actions. Recent studies revealed the impact of stress on the corticostriatal network, which comprises the $\mathrm{mPFC}$ and dorsomedial striatum (caudate in humans) and regulates goal-directed behavior. This associative network works in tandem with the adjacent sensorimotor network that links the sensorimotor cortex to the dorsolateral striatum (putamen in humans) and controls habits [88]. Recent research in rats has identified transfer of activity from the associative network to the sensorimotor network as the neural basis of the switching of instrumental behaviors from goal-directed actions into habits. This was deduced from the observation that rats undergoing chronic stress develop insensitivity to outcome value and bias their decision-making strategies towards habitual responses. The neural correlates of this switch in behavioral pattern include dendritic atrophy within the associative corticostriatal network (Figure 4a), increased volume of the dorsolateral striatum (Figure 4b), and overactivation of the sensorimotor versus associative circuits with impairments (a)

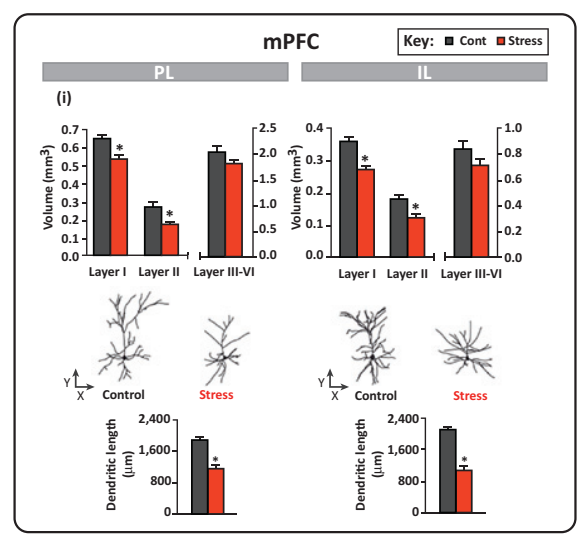

(d)

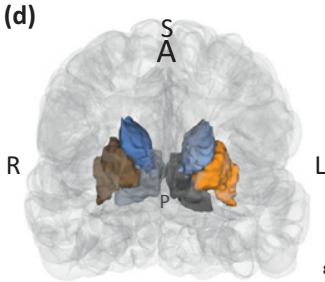

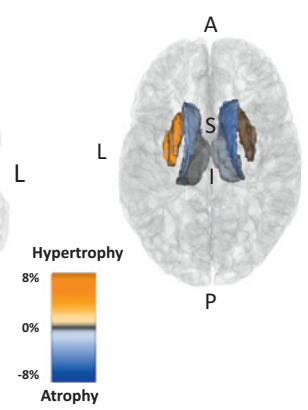

(b)

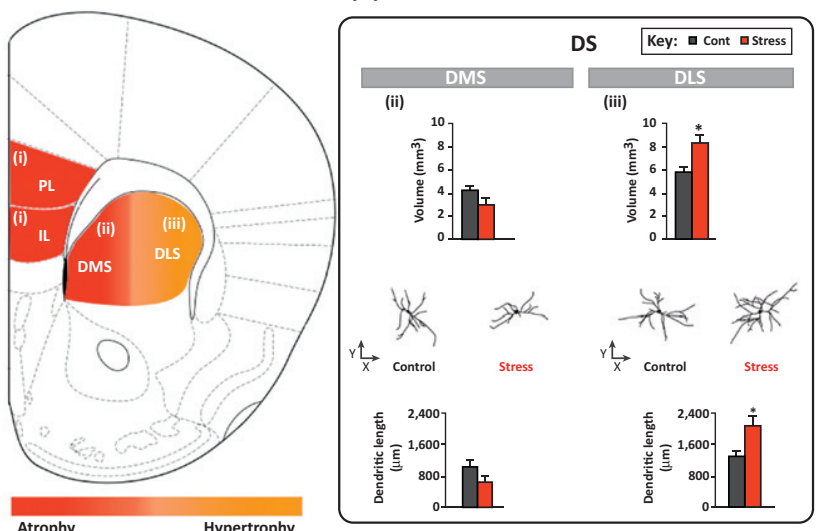

(e)

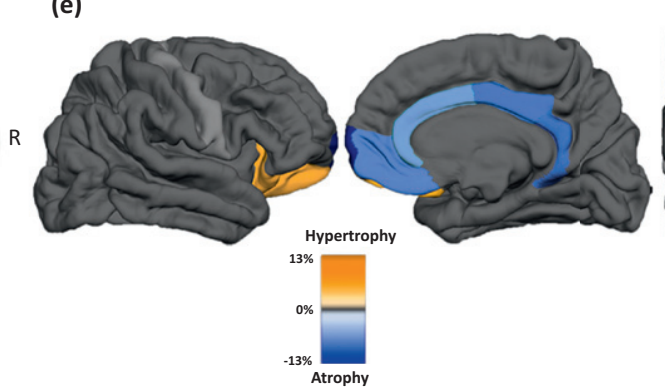

(c)

Outcome devaluation

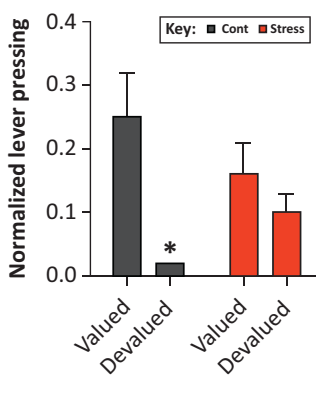

(f)

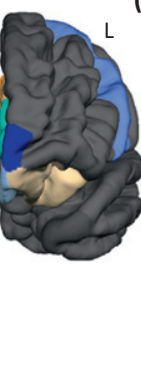

Outcome devaluation

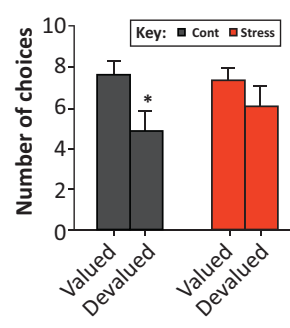

Figure 4. Stress impairs decision-making by impacting corticostriatal networks. (a,b) Comparisons between controls (Cont) and rats exposed to 21 days of chronic stress (Stress) show

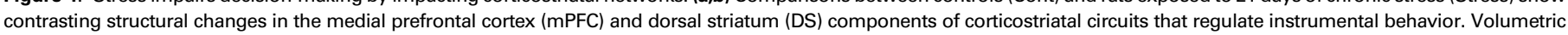
reductions and dendritic atrophy are observed in (i) the prelimbic (PL) and infralimbic (IL) cortices, as well as (ii) a trend towards dendritic atrophy in the dorsomedial striatum (DMS) these brain areas are components of the associative network that regulates goal-directed behaviors. By contrast, stress induces volumetric increases and dendritic hypertrophy in (iii)

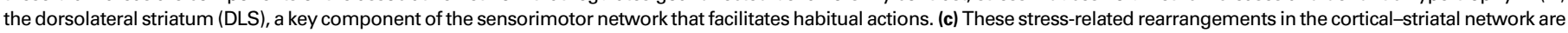
manifest behaviorally in the form of a bias towards habitual actions rather than actions that are based on an assessment of the consequences of a given behavioral strategy; habit-based actions in stressed animals are illustrated by their inability to perceive the reduction in value of a reward by satiation (outcome devaluation) in an instrumental task. (d,e) Magnetic resonance imaging in humans exposed to prolonged stress demonstrates a contrasting pattern of changes in the volume of different brain regions, with atrophy in associative network structures (such as the mPFC and caudate nuclei) and hypertrophy of the putamen (a component of the sensorimotor network) and the orbitofrontal cortex. The color changes illustrate variations in volumes for stressed subjects compared to control subjects. (f) Importantly, as for rodents, chronic stress biases instrumental actions to habits as revealed by the inability of stressed subjects to devalue the outcome. Asterisks denote statistically significant changes. Asterisks denote statistically significant changes. Adapted from (a-c) [89] and (d-f) [91]. 
in outcome devaluation (Figure 4c) [89]. The transferability of these findings to humans was recently demonstrated in studies showing that stressed subjects adopt a habit-like strategy when challenged with stimuli demanding decision-based actions [90,91]. A recent study suggested a role for mineralocorticoid receptors in mediation of the switch between memory systems [92].

As previously mentioned, the impact of stress on one behavioral domain can spread to other domains. This is because of the direct actions of stress on the substrates that regulate the different domains and the overlapping functions subserved by different anatomical areas, as well as co-dependency on intact connections between different areas [46]. First, interconnections between the hippocampal-PFC circuitry and the extended amygdala, which together regulate fear and anxiety [93], ands between the hippocampal-PFC circuitry and the mesolimbic system (i.e., ventral tegmental area and nucleus accumbens), which regulate motivation and reward [94,95], are well established. Second, interactions between different behavioral domains are exemplified by the fact that emotional state, which is accentuated under stressful conditions, is strongly linked to mood $[46,96]$ and is an important determinant of cognitive function [97]. In addition, motivation, which is clearly disturbed in depression [98], modulates cognitive functions such as learning, memory, and decision-making [99], and cognitive state influences emotional state [100].

Stress increases anxiety levels in both rodents $[46,47,101]$ and humans [102]. It is now evident that hyperanxiousness results from increased activation of the extended amygdala, most probably because of a decrease in the influence of cortical structures; this supports the view that balanced activity in cortical versus subcortical regions is essential for eliciting appropriate emotional responses [103]. Under stressful conditions, the rodent amygdala [101] and BNST [47] display dendritic hypertrophy, with activation of a reciprocal pathway between these two regions resulting in increased plasticity in excitatory synapses in the basolateral amygdala (BLA) [104]. It is thought that these morphological and neurochemical adjustments facilitate encoding of fear memory [105]. A role for BLA-PFC interactions in this process is implied by the finding that LTP in the PFC is subject to inhibition by high-frequency stimulation of the BLA but, importantly, only in the presence of stressful stimuli [106]. In this context, it is worth noting that the ventral hippocampus also contributes to the regulation of emotional behavior. Electrophysiological data suggest that the hippocampus gates information in circuits that involve the PFC and striatum (including the nucleus accumbens) [107] and acts as a hub through which information about stressful events passes before being transmitted to areas governing emotion, cognition, motivation, and the neuroendocrine response to stress. Moreover, recent work has shown that stress exerts different effects on the dorsal and ventral hippocampus; stress reduces activity of the dorsal region (implicated in cognition) and increases activity in the ventral portion (implicated in emotional behavior and control of GC secretion) [108]. Adding support to the view that the hippocampus serves to encode context and to elicit contextually appropriate responses from a number of brain regions is the recent report that anxiety levels correlate with theta coherence in the ventral hippocampus-PFC network [109]. Thus, given that the bidirectional interactions between the extended amygdala and hypothalamus are responsible for organizing a coordinated endocrine response to stress [110], the increased activity of the ventral hippocampus after stress is most likely linked to overactivation of regions of the brain that determine emotional behavior. Finally, hippocampal neurogenesis is sensitive to stress, so it seems reasonable to postulate that impaired neurogenesis may be part of the mechanism underlying the stress-induced disruption of emotional behavior [21,26-28].

\section{Concluding remarks and future directions}

The neural circuitry activated by stress is likely to be much more complex than portrayed in this brief overview. Introduction of a systems biology approach to analysis of the interdependence of the various components of this circuitry and the underlying cellular mechanisms will not only be stimulating and challenging, but will also probably help us to move closer to understanding the biological basis of a number of psychopathological states (Box 3).

In 1996, Sapolsky asked the provocative question, 'Why is stress bad for your brain?' [111]. Well, is it? Prima facie, the stress response is supposed to be adaptive. In fact,

\section{Box 3. Outstanding questions}

\section{Systematic mapping}

- Assuming that stress effects occur in a spatiotemporal pattern in which sequential responses add up to produce psychopathological features, a critical task is to determine the cellular and molecular basis of the transfer of stress effects (i.e., altered neuronal activity) between pathways.

- Given that neuronal circuits are not equally impacted by stress, it will be important to unravel how distinct neuronal networks are reorganized in response to different stressors.

\section{Cell fate and cellular phenotypes}

- What is the impact of stress-induced reductions in neurogenesis for optimal network activity and ultimately specific brain functions in health and disease?

- Given the key role of glia in brain functions, it will be important to investigate the extent to which stress influences the fate and function of non-neuronal cells in the brain.

\section{Recovery and resilience}

- Given that certain stress-induced effects are reversible, identification of the molecular correlates of the morphological and behavioral recovery seen after relief from stress may help in identifying therapeutic strategies.

- Individuals differ in their susceptibility and resistance to stress; this raises the question of whether individuals are endowed with characteristic morphofunctional response patterns that translate into better adaptive capacity and ability to resist the harmful effects of stress.

\section{Translatability of morphological findings}

- Given that morphological alterations in specific neuronal circuits and/or networks are more amenable to evaluation in living subjects than neurochemical changes are, can neuroimaging be exploited to predict the long-term consequences of stress with sufficient power to allow effective intervention? 
stress provides experience that can be called on to guide future responses and actions, but its benefits are lost when it persists or an organism's mechanisms for curtailing its physiological and behavioral responses are overwhelmed. When emphasizing the adverse and maladaptive effects of chronic stress through progressive disruption or disconnection of neural circuits, it becomes apparent that whereas stress inflicts damage on the brain, many of its actions can be spontaneously reversed by extinction of the stressor or through learning to cope with stressful challenges (e.g., through cognitive behavioral therapy, the neural mechanisms of which remain largely unknown). Current research trends in the neuroscience of stress are increasingly centered on determining the genetic and experiential factors and cognitive resources that may explain individual differences in vulnerability to the adverse effects of stress; deciphering the neuroanatomical basis of resilience to stress represents the next big challenge in this field. Fulfilling Selye's legacy requires us to recognize that stress is neither something we can or should avoid nor something that can be cured, but rather something we can be 'taught to enjoy'.

\section{Acknowledgments}

We would like to thank Antonio Pinheiro, Hugo Almeida, and José Miguel Soares for help with the illustrations. We also thank all past and present members of our laboratories for stimulating discussions. Work in our laboratories was supported by the German Academic Exchange ServiceAcções Luso-Alemãs, Fundação para a Ciência e Tecnologia (PTDC/SAUNSC/111814/2009), and European Union FP7 (SwitchBox Project, Contract 259772; Neuroendocrine Immune Networks in Ageing Scientific Network, Contract 238665).

\section{References}

1 Selye, H. (1936) A syndrome produced by diverse nocuous agents. Nature 138, 32

2 McEwen, B.S. and Giannaros, P.J. (2011) Stress- and allostasisinduced brain plasticity. Annu. Rev. Med. 62, 431-445

3 Sapolsky, R.M. et al. (2000) How do glucocorticoids influence stress responses? Integrating permissive, suppressive, stimulatory, and preparative actions. Endocr. Rev. 21, 55-89

4 de Kloet, R. et al. (2008) Corticosteroid hormones in the central stress response: quick-and-slow. Front. Neuroendocrinol. 29, 268-272

5 Sapolsky, R.M. et al. (1986) The neuroendocrinology of stress and aging, the glucocorticoid cascade hypothesis. Endocr. Rev. 7, 284301

6 Sapolsky, R.M. (1985) A mechanism for glucocorticoid toxicity in the hippocampus, increased neuronal vulnerability to metabolic insults. J. Neurosci. 5, 1228-1232

7 Chrochemore, C. et al. (2005) Direct targeting of hippocampal neurons for apoptosis by glucocorticoids is reversible by mineralocorticoid receptor activation. Mol. Psychiatry 10, 790-798

8 Landfield, P.W. et al. (1992) Mechanisms of neuronal death in brain aging and Alzheimer's disease: role of endocrine-mediated calcium dyshomeostasis. J. Neurobiol. 23, 1247-1260

9 Stein-Behrens, B. et al. (1994) Stress exacerbates neuron loss and cytoskeletal pathology in the hippocampus. J. Neurosci. 14, 53735380

$10 \mathrm{Lu}$, J. et al. (2003) Ionotropic and metabotropic glutamate receptor mediation of glucocorticoid-induced apoptosis in hippocampal cells and the neuroprotective role of synaptic $N$-methyl-D-aspartate receptors. Neuroscience $121,123-131$

$11 \mathrm{Behl}$, C. et al. (1997) Protection against oxidative stress-induced neuronal cell death - a novel role for RU486. Eur. J. Neurosci. 9, 912-920

12 Starkman, M.N. et al. (1992) Hippocampal formation volume, memory dysfunction, and cortisol levels in patients with Cushing's syndrome. Biol. Psychiatry 32, 756-765
13 Sheline, Y.I. et al. (1996) Hippocampal atrophy in recurrent major depression. Proc. Natl. Acad. Sci. U.S.A. 93, 3908-3913

14 Starkman, M.N. et al. (1999) Decrease in cortisol reverses human hippocampal atrophy following treatment of Cushing's disease. Biol. Psychiatry 46, 1595-1602

15 Frodl, T. et al. (2007) Effect of hippocampal and amygdala volumes on clinical outcomes in major depression: a 3-year prospective magnetic resonance imaging study. J. Psychiatry Neurosci. 33, 423-430

16 Colla, M. et al. (2007) Hippocampal volume reduction and HPAsystem activity in major depression. J. Psychiatr. Res. 41, 553-560

17 Sousa, N. et al. (1998) Maintenance of hippocampal cell numbers in young and aged rats submitted to chronic unpredictable stress. Comparison with the effects of corticosterone treatment. Stress 2, 237-249

18 Dranovsky, A. et al. (2011) Experience dictates stem cell fate in the adult hippocampus. Neuron 70, 908-923

19 Sahay, A. et al. (2011) Pattern separation: a common function for new neurons in hippocampus and olfactory bulb. Neuron 70, 582-588

20 Kitamura, T. et al. (2009) Adult neurogenesis modulates the hippocampus-dependent period of associative fear memory. Cell $139,814-827$

21 Bessa, J.M. et al. (2009) The mood-improving actions of antidepressants do not depend on neurogenesis but are associated with neuronal remodeling. Mol. Psychiatry 14, 764-773 739

22 David, D.J. et al. (2009) Neurogenesis-dependent and -independent effects of fluoxetine in an animal model of anxiety/depression. Neuron $62,479-493$

23 Pham, K. et al. (2003) Repeated restraint stress suppresses neurogenesis and induces biphasic PSA-NCAM expression in the adult rat dentate gyrus. Eur. J. Neurosci. 17, 879-886

24 Tichomirowa, M.A. et al. (2005) Endocrine disturbances in depression. J. Endocrinol. Invest. 28, 89-99

25 Holsboer, F. et al. (1980) Diagnostic value of dexamethasone suppression test in depression. Lancet 2, 706

26 Snyder, J.S. et al. (2011) Adult hippocampal neurogenesis buffers stress responses and depressive behaviour. Nature 476, 458-461

27 Surget, A. et al. (2011) Antidepressants recruit new neurons to improve stress response regulation. Mol. Psychiatry 16, 11177-11188

28 Airan, R.D. et al. (2007) High-speed imaging reveals neurophysiological links to behavior in an animal model of depression. Science 317, 819-823

29 Aimone, J.B. et al. (2011) Resolving new memories: a critical look at the dentate gyrus, adult neurogenesis, and pattern separation. Neuron 70, 589-596

30 Silva, A.J. et al. (2009) Molecular and cellular approaches to memory allocation in neural circuits. Science 326, 391-395

$31 \mathrm{Yu}, \mathrm{S}$. et al. (2010) Depletion of the neural precursor cell pool by glucocorticoids. Ann. Neurol. 67, 21-30

$32 \mathrm{Yu}$, S. et al. (2011) Glucocorticoid regulation of astrocytic fate and function. PLoS ONE 6, e22419

33 Banasr, M. et al. (2007) Chronic unpredictable stress decreases cell proliferation in the cerebral cortex of the adult rat. Biol. Psychiatry 62, 496-504

34 Banasr, M. et al. (2010) Glial pathology in an animal model of depression: reversal of stress-induced cellular, metabolic and behavioral deficits by the glutamate-modulating drug riluzole. Mol. Psychiatry 15, 501-511

35 Halassa, M.M. and Haydon, P.G. (2010) Integrated brain circuits: astrocytic networks modulate neuronal activity and behavior. Annu. Rev. Physiol. 17, 335-355

36 Chalifoux, J.R. and Carter, A.G. (2011) Glutamate spillover promotes the generation of NMDA spikes. J. Neurosci. 31, 16435-16446

37 Rajkowska, G. (2000) Postmortem studies in mood disorders indicate altered numbers of neurons and glial cells. Biol. Psychiatry 48, 766-777

38 Woolley, C.S. et al. (1990) Exposure to excess glucocorticoids alters dendritic morphology of adult hippocampal pyramidal neurons. Brain Res. 531, 225-231

39 Sousa, N. et al. (2000) Reorganization of the morphology of hippocampal neurites and synapses after stress-induced damage correlates with behavioral improvement. Neuroscience 97, 253-266

40 van Eekelen, J.A. et al. (1991) Postnatal ontogeny of mineralocorticoid and glucocorticoid receptor gene expression in regions of the rat teland diencephalon. Dev. Brain Res. 61, 33-43 
41 Patel, P.D. et al. (2008) Stress-induced changes in corticosteroid receptor expression in primate hippocampus and prefrontal cortex. Psychoneuroendocrinology 33, 360-367

42 Groeneweg, F.L. et al. (2012) Mineralocorticoid and glucocorticoid receptors at the neuronal membrane, regulators of nongenomic corticosteroid signalling. Mol. Cell. Endocrinol. 350, 299-309

43 Pacak, K. and Palkovits, M. (2001) Stressor specificity of central neuroendocrine responses: implications for stress-related disorders. Endocr. Rev. 22, 502-548

44 Joels, M. and Baram, T. (2009) The neuro-symphony of stress. Nat. Rev. Neurosci. 10, 459-466

45 Ulrich-Lai, Y.M. and Herman, J.P. (2009) Neural regulation of endocrine and autonomic stress responses. Nat. Rev. Neurosci. 10, 397-409

46 Bessa, J.M. et al. (2009) A trans-dimensional approach to the behavioral aspects of depression. Front. Behav. Neurosci. 3, 1

47 Pêgo, J.M. et al. (2008) Dissociation of the morphological correlates of stress-induced anxiety and fear. Eur. J. Neurosci. 27, 1503-1516

48 Dalley, J.W. et al. (2004) Prefrontal executive and cognitive functions in rodents, neural and neurochemical substrates. Neurosci. Biobehav. Rev. 28, 771-784

49 Popoli, M. et al. (2011) The stressed synapse: the impact of stress and glucocorticoids on glutamate transmission. Nat. Rev. Neurosci. 13, $22-37$

50 Meaney, M.J. et al. (1985) $\left[{ }^{3} \mathrm{H}\right]$ dexamethasone binding in the limbic brain of the fetal rat. Brain Res. 355, 179-185

51 Figueiredo, H.F. et al. (2003) The medial prefrontal cortex differentially regulates stress-induced c-fos expression in the forebrain depending on type of stressor. Eur. J. Neurosci. 18, 2357-2364

52 Sullivan, R.M. and Gratton, A. (2002) Prefrontal cortical regulation of hypothalamic-pituitary-adrenal function in the rat and implications for psychopathology, side matters. Psychoneuroendocrinology 27, 99114

53 Sapolsky, R.M. et al. (1985) The development of the glucocorticoid receptor system in the rat limbic brain. III. Negative-feedback regulation. Brain Res. 350, 169-173

54 Mizoguchi, K. et al. (2003) Chronic stress attenuates glucocorticoid negative feedback, involvement of the prefrontal cortex and hippocampus. Neuroscience 119, 887-897

55 Radley, J.J. et al. (2004) Chronic behavioral stress induces apical dendritic reorganization in pyramidal neurons of the medial prefrontal cortex. Neuroscience 125, 1-6

56 Cerqueira, J.J. et al. (2005) Corticosteroid status influences the volume of the rat cingulate cortex - a magnetic resonance imaging study. J. Psychiatr. Res. 39, 451-460

57 Wellman, C.L. (2001) Dendritic reorganization in pyramidal neurons in medial prefrontal cortex after chronic corticosterone administration. J. Neurobiol. 49, 245-253

58 Cook, C.S. and Wellman, C.L. (2004) Chronic stress alters dendritic morphology in rat medial prefrontal cortex. J. Neurobiol. 60, 236-248

59 Radley, J.J. et al. (2006) Repeated stress induces dendritic spine loss in the rat medial prefrontal cortex. Cereb. Cortex 16, 313-320

60 Cerqueira, J.J. et al. (2007) Specific configuration of dendritic degeneration in pyramidal neurons of the medial prefrontal cortex induced by differing corticosteroid regimens. Cereb. Cortex 17, 19982006

61 Radley, J.J. et al. (2008) Repeated stress alters dendritic spine morphology in the rat medial prefrontal cortex. J. Comp. Neurol. $507,1141-1150$

62 Shansky, R.M. et al. (2009) Stress-induced dendritic remodeling in the prefrontal cortex is circuit specific. Cereb. Cortex 19, 2479-2484

63 Shansky, R.M. et al. (2010) Estrogen promotes stress sensitivity in a prefrontal cortex-amygdala pathway. Cereb. Cortex 20, 2560-2567

64 Golwater, D.S. (2009) Structural and functional alterations to rat medial prefrontal cortex following chronic restraint stress and recovery. Neuroscience 164, 798-808

65 Perez-Cruz, C. et al. (2007) Morphology of pyramidal neurons in the rat prefrontal cortex: lateralized dendritic remodeling by chronic stress. Neural Plast. 2007, 46276

66 Perez-Cruz, C. et al. (2009) Hemispheric differences in basilar dendrites and spines of pyramidal neurons in the rat prelimbic cortex: activity- and stress-induced changes. Eur. J. Neurosci. 29, 738-747
67 MacLullich, A.M. et al. (2006) Smaller left anterior cingulate cortex volumes are associated with impaired hypothalamic-pituitaryadrenal axis regulation in healthy elderly men. J. Clin. Endocrinol. Metab. 91, 1591-1594

68 Goel, V. and Dolan, R.J. (2004) Differential involvement of left prefrontal cortex in inductive and deductive reasoning. Cognition 93, B109-B121

69 Reverberi, C. et al. (2005) Specific impairments of rule induction in different frontal lobe subgroups. Neuropsychologia 43, 460-472

70 Corballis, P.M. (2003) Visuospatial processing and the righthemisphere interpreter. Brain Cogn. 53, 171-176

71 Goel, V. et al. (2000) Dissociation of mechanisms underlying syllogistic reasoning. Neuroimage 12, 504-514

72 Drevets, W.C. et al. (2002) Functional anatomical correlates of antidepressant drug treatment assessed using PET measures of regional glucose metabolism. Eur. Neuropsychopharmacol. 12, 527544

73 Goldapple, K. et al. (2004) Modulation of cortical-limbic pathways in major depression: treatment-specific effects of cognitive behavior therapy. Arch. Gen. Psychiatry 61, 34-41

74 Cerqueira, J.J. et al. (2007) The prefrontal cortex as a key target of the maladaptive response to stress. J. Neurosci. 27, 2781-2787

75 Brown, S.M. et al. (2005) Mild, short-term stress alters dendritic morphology in rat medial prefrontal cortex. Cereb. Cortex 15, 17141722

76 Izquierdo, A. et al. (2006) Brief uncontrollable stress causes dendritic retraction in infralimbic cortex and resistance to fear extinction in mice. J. Neurosci. 26, 5733-5738

77 Sotiropoulos, I. et al. (2011) Stress acts cumulatively to precipitate Alzheimer's disease-like tau pathology and cognitive deficits. $J$. Neurosci. 31, 7840-7847

78 Lee, Y.A. et al. (2011) Dorsal-ventral distinction of chronic stressinduced electrophysiological alterations in the rat medial prefrontal cortex. Neuroscience 183, 108-120

79 Benchenanne, K. et al. (2010) Coherent theta oscillations and reorganization of spike timing in the hippocampal-prefrontal network upon learning. Neuron 66, 921-936

80 Jones, M.W. and Wilson, M.A. (2005) Theta rhythms coordinate hippocampal-prefrontal interactions in a spatial memory task. PLoS Biol. 3, e402

81 Wilber, A.A. et al. (2011) Chronic stress alters neural activity in medial prefrontal cortex during retrieval of extinction. Neuroscience 174, 115-131

82 Radley, J.J. et al. (2005) Reversibility of apical dendritic retraction in the rat medial prefrontal cortex following repeated stress. Exp. Neurol. 196, 199-203

83 Bloss, E.B. et al. (2010) Interactive effects of stress and aging on structural plasticity in the prefrontal cortex. J. Neurosci. 30, 67266731

84 Castrén, E. (2005) Is mood chemistry? Nat. Rev. Neurosci. 6, 241-246

85 Krugers, H.J. et al. (2010) Stress hormones and AMPA receptor trafficking in synaptic plasticity and memory. Nat. Rev. Neurosci. $11,675-681$

86 Sandi, C. (2011) Glucocorticoids act on glutamatergic pathways to affect memory processes. Trends Neurosci. 34, 165-176

87 Martin, K.P. and Wellman, C.L. (2011) NMDA receptor blockade alters stress-induced dendritic remodeling in medial prefrontal cortex. Cereb. Cortex 21, 2366-2373

88 Yin, H.H. and Knowlton, B.J. (2006) The role of the basal ganglia in habit formation. Nat. Rev. Neurosci. 7, 464-476

89 Dias-Ferreira, E. et al. (2009) Chronic stress causes frontostriatal reorganization and affects decision-making. Science 325, 621625

90 Schwabe, L. and Wolf, O.T. (2009) Stress prompts habit behavior in humans. J. Neurosci. 29, 7191-7198

91 Soares, J.M. et al. (2012) Stress-induced changes in human decisionmaking are reversible. Transl. Psychiatry 2, e131

92 Schwabe, L. et al. (2010) Corticosteroids operate as a switch between memory systems. J. Cogn. Neurosci. 22, 1362-1372

93 Ottersen, O.P. (1982) Connections of the amygdala of the rat. IV: corticoamygdaloid and intraamygdaloid connections as studied with axonal transport of horseradish peroxidase. J. Comp. Neurol. 205, $30-48$ 
94 French, S.J. and Totterdell, S. (2002) Hippocampal and prefrontal cortical inputs monosynaptically converge with individual projection neurons of the nucleus accumbens. J. Comp. Neurol. 446, 151-165

95 French, S.J. and Totterdell, S. (2003) Individual nucleus accumbensprojection neurons receive both basolateral amygdala and ventral subicular afferents in rats. Neuroscience 119, 19-31

96 Ressler, K.J. and Mayberg, H.S. (2007) Targeting abnormal neural circuits in mood and anxiety disorders, from the laboratory to the clinic. Nat. Neurosci. 10, 1116-1124

97 McGaugh, J.L. (2006) Make mild moments memorable, add a little arousal. Trends Cogn. Sci. 10, 345-347

98 Scheurich, A. et al. (2008) Experimental evidence for a motivational origin of cognitive impairment in major depression. Psychol. Med. 38, 237-246

99 Locke, H.S. and Braver, T.S. (2008) Motivational influences on cognitive control, behavior, brain activation, and individual differences. Cogn. Affect. Behav. Neurosci. 8, 99-112

100 Bechara, A. et al. (2000) Characterization of the decision-making deficit of patients with ventromedial prefrontal cortex lesions. Brain 123, 2189-2202

101 Mitra, R. et al. (2005) Stress duration modulates the spatiotemporal patterns of spine formation in the basolateral amygdala. Proc. Natl. Acad. Sci. U.S.A. 102, 9371-9376

102 Martin, E.I. et al. (2009) The neurobiology of anxiety disorders: brain imaging, genetics, and psychoneuroendocrinology. Psychiatr. Clin. North Am. 32, 549-575

103 Davis, M. et al. (1997) Roles of the amygdala and bed nucleus of the stria terminalis in fear and anxiety measured with the acoustic startle reflex. Possible relevance to PTSD. Ann. N. Y. Acad. Sci. 821, 305-331

104 Kavushansky, A. and Richter-Levin, G. (2006) Effects of stress and corticosterone on activity and plasticity in the amygdala. J. Neurosci. Res. 84, 1580-1587
105 Rodriguez Manzanares, P.A. et al. (2005) Previous stress facilitates fear memory, attenuates GABAergic inhibition, and increases synaptic plasticity in the rat basolateral amygdala. J. Neurosci. 25, 8725-8734

106 Maroun, M. and Richter-Levin, G. (2003) Exposure to acute stress blocks the induction of long-term potentiation of the amygdalaprefrontal cortex pathway in vivo. J. Neurosci. 23, 4406-4409

107 O'Donnell, P. and Grace, A.A. (1995) Synaptic interactions among excitatory afferents to nucleus accumbens neurons, hippocampal gating of prefrontal cortical input. J. Neurosci. 15, 3622-3639

108 Maggio, N. and Segal, M. (2009) Differential modulation of long-term depression by acute stress in the rat dorsal and ventral hippocampus. J. Neurosci. 29, 8633-8638

109 Adhikari, A. et al. (2010) Synchronized activity between the ventral hippocampus and the medial prefrontal cortex during anxiety. Neuron 65, 257-269

110 Bhatnagar, S. et al. (2004) Regulation of chronic stress-induced changes in hypothalamic-pituitary-adrenal activity by the basolateral amygdala. Ann. N. Y. Acad. Sci. 1032, 315-319

111 Sapolsky, R.M. (1996) Why stress is bad for your brain. Science 273, 749-750

112 Liston, C. et al. (2006) Stress-induced alterations in prefrontal cortical dendritic morphology predict selective impairments in perceptual attentional set-shifting. J. Neurosci. 26, 7870-7874

$113 \mathrm{Di}$, S. et al. (2003) Nongenomic glucocorticoid inhibition via endocannabinoid release in the hypothalamus: a fast feedback mechanism. J. Neurosci. 23, 4850-4857

114 Karst, H. et al. (2005) Mineralocorticoid receptors are indispensable for nongenomic modulation of hippocampal glutamate transmission by corticosterone. Proc. Natl. Acad. Sci. U.S.A. 102, 19204-19207

115 Riedemann, T. et al. (2010) Corticosteroids: way upstream. Mol. Brain 3,2 